\title{
Ueber das Saponin aus den Blättern von Trevesia sundaica Miq. ${ }^{2}$.
}

Von J. Fliering a ${ }^{2}$ ).

(Eingegangen den 28. I. 1911.)

\section{Darstellung des Rohsaponins.}

Das für diese Arbeit verwendete Material stammte aus Buitenzorg (Java). Ueber das Saponin der Blätter ist bisher noch keine Untersuchung publiziert; über das Saponin der Rinde hat B o o r s $\mathrm{m} \mathrm{a}^{3}$ ) nur eine vorläufige Untersuchung angestellt.

Mit ziemlich gutem Erfolge kann das Rohsaponin der Blätter dargestellt werden nach der Methylalkoholmethode4). Da es sich jedoch ergab, daß das Saponin durch Sättigung der Lösung mit Ammoniumsulfat ${ }^{5}$ ) völlig ausgesalzen werden kann und dasselbe überdies in absolutem Alkohol löslich ist, wodurch das Ammoniumsulfat wieder leicht entfernt werden kann, so wurde das Saponin an Stelle der lange dauernden Dialyse, welche bei der Methylalkoholmethode erforderlich ist, mit Ammoniumsulfat ausgefällt.

Zur Darstellung des Rohsaponins wurden $2 \mathrm{~kg}$ der getrockneten, pulverisierten Blätter während längerer Zeit mit starkem Spiritus perkoliert; nachher wurden sie wiederholt mit dem Spiritus ausgekocht. Nachdem der Spiritus unter vermindertem Drucke abdestilliert war, wurde die Trockensubstanz mit stark verdünntem Spiritus aufgenommen. Diese Lösung wurde wiederholt mit Aother ausgeschüttelt, um Pflanzenwachs und Chlorophyll zu beseitigen. Hierauf wurde sie teilweise eingedampft, zur Beseitigung des Aethers und des Alkohols, und mit Wasser verdünnt. Aus dieser Lösung wurde dann das Saponin durch Sättigung mit Ammoniumsulfat ausgefällt. Das Saponin wurde hierauf noch einmal in Wasser gelöst und wieder ausgesalzen. Der braune, gut zusammenballende, leicht zerreibliche Niederschlag wurde wiederholt mit starkem Spiritus ausgekocht, wobei das Saponin sich löste, während braune Sub-

1) M i q u \& l, Flora von Indie, I., S. 747.

2) Autoreferat einer Dissertation, Utrecht 1910.

3) Mededeelingen uit's Lands-Plantentuin 52, 77 (1902).

4) B o o r s m a, Mbid. 52, 30 (1902).

5) Ko bert, Beiträge zur Kenntnis der Saponinsubstanzen, i. 20 (1904).

Aroh. d. Pharm. Cexxxxix. Bds. 3. Haft. 
stanzen und der größte Teil des Ammoniumsulfats zurückblieben. Die Lösung wurde schließlich bei niedriger Temperatur eingedampft und ließ ca. $100 \mathrm{~g}$ braunes Rohsaponin zurück $(5 \%$ der getrockneten Blätter).

Das Saponin wird mit Ammoniumsulfat so gut wie vollkommen gefällt. Wird das nach der Fällung erhaltene Filtrat mit dem zweifachen Volum Salzsäure von $12,5 \%$ gekocht, so entsteht nur eine geringe, braune, nicht flockige Trübung, wogegen eine sehr verdünnte Saponinlösung (z. B. $1: 2000$ ) unter diesen Umständen einen flockigen Niederschlag von Sapogenin gibt.

Durch die Ausfällung mit Ammoniumsulfat werden hygroskopische Substanzen (u. a. Zucker) beseitigt; das Rohsaponin wird an der Luft nicht mehr feucht.

Die Verwendung des Ammoniumsulfats zur Ausfällung der Saponine (nach K o b e r t) ist nicht ganz einwandfrei. Beim Eindampfen einer Ammoniumsulfatlösung erhält sie saure Reaktion. Nach dem Eintrocknen einer Saponinlösung, die etwas Ammoniumsulfat enthält, ist deshalb in dem Rückstande etwas freie Sohwefelsäure enthalten. Weil meine Saponinsubstanzen auch völlig durch Sättigung der Lösung mit Magnesiumsulfat ausgefällt werden konnten, habe ich nachher zur Ausfällung der Saponine immer chlorfreies Magnesiumsulfat verwendet, welchem dieses Uebel nicht anhaftet.

\section{Eigenschaften des Rehseponins.}

Daß hier wirklich eine Saponinsubstanz vorliegt, ergibt sich aus den folgenden Eigenschaften.

Das Rohsaponin löst sich klar in Wasser. Beim Schütteln der verdünnten Lösungen bildet sich ein starker Sehaum. Die Substanz hat glykosidische Natur; beim Erhitzen mit Säure wird sie gespalten in Zucker und einen in Wasser unlöslichen Stoff.

Auch ist sie hämolytisch. Wunde in einem Gemisch, erhalten durch Verdünnung von $\mathbf{1} \mathbf{g}$ defibriniertem Rinderblut mit Kochsalzlösung von 0,8\% zu $100 \mathrm{ccm}$, das Saponin in dem Verhältnis 1: 500 gelöst, so war es in 3 Mịnuten klar und lackfarben; mit Saponin $1: 1000$ in 10 Minuten.

Abweichend von den meisten Saponinsubstanzen löst sich das Rohsaponin leicht in Spiritus von $90 \%$, auch in hoißem absolutem Alkohol; weniger gut in kaltem absolutem Alkohol.

Neutrales Bleiacetat gibt in der wässerigen Lösung einen braunen Niederschlag, in welchem sich jedoch kein Saponin vorfand. Durch basisches Bleiacetat werden wohl Saponinsubstanzen gefällt, 
in verdünnter Lösung jedoch erst beim Erhitzen. Je stärker die Basizität des Bleiacetats ist, desto mehr wird gefällt. Bleiessig (halbbasisches Bleiacetat) fällt also mehr als ein Gemisch von Bleiessig mit neutralem Bleiacetat und weniger als einfach basisches Bleiacetat; erst durch Bleiessig + Ammoniak wird alles gefällt.

Beim Erhitzen mit Bleiessig werden auch die braunen und die (sauren) gelben Substanzen des Rohsaponins gefällt; aus dem Filtrat kann dann ein nur schwach gefärbtes Saponin erhalten werden.

\section{Bohandlung mit Magnesiumoxydhydrat.}

Auch nach der Magnesiamethode kann eine schwach gefärbte Fraktion des Rohsaponins erhalten werden. Wird jedoch in der gewöhnlichen Weise die Saponinlösung mit Magnesiumoxyd eingetrocknet und der Rückstand mit Alkohol ausgekocht, so tritt teilweise Spaltung ein. Deshalb wurde die Methode so abgeändert, daß das Eintrocknen und das Auskochen umgangen wurden.

Statt des Magnesiumoxyds wurde Magnesiumoxydhydrat verwendet, welches sich bildet, wenn man das Oxyd mit Wasser mischt. Das Hydrat wird abgesogen und durch wiederholtes Anreiben mit Alkohol und Absaugen entwässert. Mit diesem alkoholfeuchten Hydrat wurde die weingeistige Lösung des Rohsaponins geschüttelt; es zerteilte sich zu einer Paste, welche nebst einem Teile des Saponins die braunen und die (sauren) gelben Substanzen band. Durch Eindampfen des Filtrats wurde alsdann ein schwach g e 1 b e s S a p o n i n erhalten. Zur Darstellung eines nur wenig gefärbten Saponins wurde dieses Verfahren der Bleiessigbehandlung vorgezogen, weil es mir leichter und sicherer dünkte.

Die Substanzen, welche durch das Magnesiumoxydhydrat gebunden sind, können zurückgewonnen werden durch Lösen desselben in verdünnter Schwefelsäure, Aussalzen mit Magnesiumsulfat und Auskochen des Niederschlages mit Alkohol, wodurch ein grünliches Saponin gelöst wird. Bei der Auflösung des Magnesiumoxydhydrats in Sohwefelsäure ist Sorge zu tragen, daß die Lösung schwach alkalisch bleibt. Hierdurch bleiben beim Aussalzen braune Substanzen gelöst, welohe in saurer Lösung mitgefällt werden.

Aus dem grünlichen Saponin kann durch neue Behandlung mit Magnesiumoxydhydrat noch etwas gelbliches Saponin erhalten werden. Aus dem stark gelben Hydrat kann wieder ein $\mathrm{g} r$ ü n li ches $S$ a p on in gewonnen werden.

Die Ausbeute aus $70 \mathrm{~g}$ Rohsaponin betrug etwra $40 \mathrm{~g}$ gelbes und $10 \mathrm{~g}$ grünes Saponin. 
Das grüne und das gelbe Saponin sind durch die folgenden Eigenschaften unterschieden.

Das grüne Saponin ist hämolytisch; das Blutgenisch $1: 100$ wurde mit Saponin 1:6000 in 16 Minuten völlig hämolysiert. Das gelbe Saponin ist dagegen kaum hämolytisch.

Mit Bleiessig erhitzt, gab das grüne Saponin einen starken gelben, das gelbe Saponin nur einen geringen, kaum gefärbten Niederschlag.

Das grüne Saponin ist nicht weiter untersucht. Die folgende Untersuchung bezieht sich nur auf das gelbe Saponin.

\section{Das golbe Saponin.}

Aus dem gelben Saponin konnte eine von der (neutralen) gelben Substanz befreite Fraktion erhalten werden durch wioderholte Fällung aus einer konzentrierten weingeistigen Lösung mit dem gleichen Volum Aether. Aus dem Trockenrückstande der dabei erhaltenen alkoholisch-ätherischen Lösungen konnte noch eine ähnliche Fraktion erhalten werden durch wiederholte Fällung aus einem kleineren Volum Spiritus mit 1 Volum Aether. Die beiden Fraktionen bilden zusammen die F r a $\mathrm{kt}$ i o $\mathrm{n} 1$.

In derselben Weise konnten aus den jedesmal erhaltenen Trockenrückständen der alkoholisch-ätherischen Lösungen noch zwei Fraktionen fast ohne gelbe Substanz erhalten werden, die eine (Fraktion 2) durch wiederholte Fällung der konzentrierten weingeistigen Lösung mit dem zweifachen Volum Aether, die andere (F r a k t i o n 3) durch Fällung mit dem siebenfachen Volum Aether.

Die schwach bräunlichen Fraktionen wurden durch Schütteln der weingeistigen Lösungen mit Magnesiumoxydhydrat teilweise, und nachher durch Kochen in absolut-alkoholischer Lösung mit Blutkohle fast völlig entfärbt.

Zur weiteren Reinigung wurden die Fraktionen noch einige Mal aus wässeriger Lösung mit Magnesiumsulfat ausgesslzen. Dem Niederschlage wurde das Saponin mit Spıritus entzogen. Nach dem Eindampfen dieser Lösungen wurde der Rückstand unter Erwärmung gelöst in einem Gemische gleicher Teile absoluten Alkohols und Chloroforms, wobei die Reste des Magnesiumsulfats ungelöst zurückblieben. Aus den filtrierten Lösungen wurden die Fraktionen mit viel Aether gefällt und nachher bei niedriger Temperatur getrocknet.

Aus $40 \mathrm{~g}$ gelbem Saponin betrug die Ausbeute etwa $18 \mathrm{~g}$ der Fraktion 1, 2,5 $\mathrm{g}$ der Fraktion 2 und $3 \mathrm{~g}$ der Fraktion 3. Diese Substanzen enthielten nur wenig Asche (weniger als 0,25\%), und gaben 
in einer Lösung von $5 \%$ keine Reaktion auf Schwefelsïure. Sie waren amorph.

Am Ende der Fraktionierung des gelben Saponins restierte eine Lösung, welche auf einen Teil Spiritus sieben Teile Aether enthielt. Diese Lösung wurde mit Wasser geschüttelt, wodurch sich zwei Schichten bildeten. Die ätherische Schicht ließ beim Eindampfen eine wachsartige Substanz zurück, welche nicht weiter untersucht wurde. Beim teilweisen Eindampfen der wässerigen Schicht schied sich eine weiße Substanz aus. Zur Reinigung dieses Stoffes wurde seine weingeistige Lösung mit Wasser gemischt und teilweise eingedampft (zur Beseitigung des Spiritus), wodurch die Substanz sich wieder ausschied (F r a k t i o n 4).

Dieser Stoff ist kaum löslich in Wasser, leicht löslich in Alkohol und Eisessig. Wurde eine Lösung in Eisessig bis zur Trübung mit Wasser verdünnt, so schieden sich, beim Stehen an der Luft, mikroskopische Nadeln aus, gewöhnlich zu kugeligen Drusen vereint.

Die Fraktion 4 hatte ebenso wie die anderen Fraktionen glykosidische Natur.

\section{Elgenschaften der Fraktionen des golben Saponins.}

Beim $\mathrm{Erh}$ it z e n fingen die Fraktionen bei ca. $140^{\circ}$ sich zu bräunen an; bei $215-220^{\circ}$ verwandelten sie sich in teerige Massen.

In kaltem $\mathrm{W}$ a s s e $\mathrm{r}$ sind die Fraktionen 1, 2 und 3 gut löslich. Aus einer konzentrierten Lösung der Fraktion 3 scheidet sich beim Erhitzen eine gallertartige Substanz aus, die sich beim Abkühlen wieder löst. Die Fraktion 4 war kaum löslich in Wasser; gemischt mit vier Teilen der Fraktion 1 löste sie sich jedoch klar in Wasser, auch in der Hitze.

In kaltem absolutem A $\mathrm{l} \mathrm{k} \mathrm{o} \mathrm{h} \mathrm{o} \mathrm{I} \mathrm{sind} \mathrm{die} \mathrm{Fraktionen} 3$ und 4 leicht löslich, die Fraktionen 1 und 2 viel weniger, aber gut beim Erhitzen. In Spiritus von $70 \%$ sind alle Fraktionen, auch die Fraktion 4, gut löslich.

Mit M a g n e s i u m s u l a t kann die Fraktion 3 viel leichter ausgesalzen werden als die Fraktionen 1 und 2 . Um $2 \mathrm{ccm}$ einer wässerigen Lösung von $5 \%$ bleibend zu trüben, waren bei $12,5^{\circ}$ nötig bei der Fraktion 1: 6,2 ccm, bei der Fraktion 2: $5,15 \mathrm{ccm}$ und bei der Fraktion 3: 1,0 ccm einer gesättigten Magnesiumsulfatlösung.

Wurden die Lösungen, welche mit Magnesiumsulfat bis zur anfangenden Trübung versetzt waren, auch nur wenig erwärmt, so schied sich ein Teil des Saponins gallertartig aus, wie dieses beim 
Erwärmen einer konzentrierten Lösung der Fraktion 4 auch ohne Magnesiumsulfat schon stattfindet.

Auch das spezifische Drehungs verm ögen der Fraktionen ist verschieden. Berechnet auf bei $105-110^{\circ}$ bis zum konstanten Gewichte getrocknetes Saponin wurde in wässeriger Lösung erhalten für die Fraktion $1[\alpha]_{\mathrm{D}}^{10}=-13,2^{0}$, für die Fraktion 2: $-4,6^{0}$, für die Fraktion $3:+30,3^{0}$ und für die Fraktion 4 (in Spiritus von $70 \%$ ): $+37^{\circ}$.

Die Fraktion 1 wurde durch Lösen in heißem absolutem Alkohol und Abkühlen, wobei ein Teil des Saponins sich wieder abschied, in zwei Fraktionen zerlegt. Das Saponin aus der Lösung hatte ein spezifisches Drehungsvermögen von $-10,5^{0}$, das gefällte Saponin von $-14,1^{\circ}$. Hieraus ergibt sich, daß die Fraktion 1 keine einfache Substanz ist.

Mit starker S c hw ef e l s ä u r e übergossen, färbten sich die Substanzen schwach orangegelb und lösten sich allmählich mit dieser Farbe in der Schwefelsäure, welche an der Luft vom Rande aus purpurfarben wurde. Nach einiger Zeit verblassen die Lösungen, indem sich ein bei den verschiedenen Fraktionen etwas abweichend gefärbter Niederschlag bildet.

Mit $\mathrm{E}$ i s e n c hlorid gaben die wässerigen Lösungen der Fraktionen 1, 2 und 3 keine Farbenreaktion.

Mit der Fehling'schen Ku pferlösung werden die Lösungen der Fraktionen 1, 2 und 3 größtenteils gefällt. Beim Erhitzen des Filtrats tritt eine schwache Reduktion ein, welche wenigstens teilweise wohl herrührt von dem Zucker, der durch die stark alkalische Lösung aus dem gelöst gebliebenen Saponin abgespalten wird.

Mit gesättigtem B a r y w a s s e r gab nur die Fraktion 3 einen in Wasser löslichen Niederschlag.

Mit neutralem B l e i a c e t a t gab nur die Lösung der Fraktion 3 eine geringe Trübung. Beim Erhitzen mit Bleiessig gaben nur die Fraktion 3 und die Fraktion 4, mittels der Fraktion 1 in Wasser gelöst, einen Niederschlag. Mit einbasischem Bleiacetat gaben auch die Fraktionen 1 und 2 in nicht zu sehr verdünnten Lösungen beim Erhitzen Niederschläge. Mit Bleiessig + Ammoniak tritt völlige Fällung ein.

Beim lange dauernden Erhitzen der Fraktionen 1 und 2 mit Bleiessig bildet sich wohl ein Niederschlag, vielleicht zufolge der Spaltung des Saponins durch den alkalischen Bleiessig.

Auch wurde die elementaranalytische Zusammensetzung der Fraktionen verglichell. Sic enthalten nur wenig Asche. In $25 \mathrm{mg}$ 
der Fraktionen 1, 2 und 4 ließ sich mittels der Reaktion von $\mathrm{Ca}$ stellan ${ }^{1}$ ) kein Stickstoff nachweisen; die Fraktion 3 gab jedoch eine schwache Reaktion. Das zur Analyse benutzte Saponin wurde bei $105-110^{\circ}$ bis zum konstanten Gewichte getrocknet, wobei es sich ein wenig bräunte.

Die folgenden Zusammensetzungen sind jedesmal berechnet aus zwei genügend uibereinstimmenden Analysen.

Die Fraktion 1 enthält 7,91\% H; 57,81\% C; 34,28\% 0 .

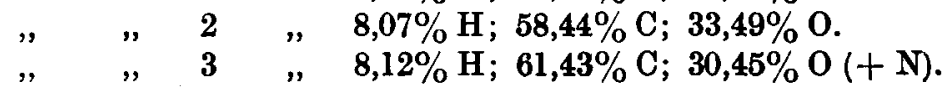

Der Kohlenstoff- und Wasserstoffgehalt steigt daher von der ersten bis zur dritten Fraktion.

Wegen Substanzmangel wurde die Fraktion 4 nicht analysiert. Die Fraktion 1 ist nicht $\mathrm{h} \ddot{\mathrm{a}} \mathrm{m} \mathrm{ol} \mathrm{y} \mathrm{t}$ i $\mathrm{s} \mathrm{ch}$, die Fraktion 2 und 3 kaum. Die Fraktion 4, mittels 5 Teilen der Fraktion 1 gelöst, war wohl hämolytisch. Das Blutgemisch $1: 100$ wurde in 24 Stunden durch die Fraktion 4, $1: 250$ völlig hämolysiert, durch die Lösung I : 500 nicht mehr.

Diese Substanzen haben dic Eigentümlichkeit, daß die eine E in $f l u B$ hat auf die Eigenschaften der anderen.

Ein Beispiel hierfür ist die Wasserlöslichkeit der Fraktion 4 mittels der Fraktion 1.

Eine Lösung der Fraktion 3, in welcher eine Menge der Fraktion 1 gelöst ist, bedarf mehr Magnesiumsulfat zur anfangenden Trübung als dieselbe Lösung ohne die Fraktion 1.

Die Lösung eines Gemisches von einem Teile der Fraktion 3 und vier Teilen der Fraktion 1 gibt mit Bleiessig keinen Niederschlag mehr.

Sogar wird das hämolytische Vermögen einer Lösung des grünen Saponins beträchtlich herabgesetzt (oder wenigstens verzögert) wenn darin eine Menge der Fraktion 1 gelöst wird.

Diese gegenseitige Einwirkung erschwert die Reindarstellung der verschiedenen Substanzen.

\section{Die Fraktion I des golben Saponins.}

Enthielt die Fraktion 1 hauptsächlich nur eine Substanz, mit kleinen Quantitäten der Fraktionen 3 und 4 gemischt, so mußten diese beigemengten Substanzen durch wiederholte Fällung aus Alkohol beseitigt werden können.

1) Chem. Contralbl., Jahrg. 1905, I., s. 45. 
$\mathrm{Zu}$ diesem Zwecke wurde die Fraktion 1 in dem zehnfachen Volum heißen absoluten Alkohols gelöst. Beim Abkühlen schied sich der größte Teil des Saponins in amorphem Zustande an der Wand des Kolbens ab. Aus der abgegossenen Lösung wurde das Saponin mit dem zehnfachen Volum Aether gefällt. Nachher wurde es im Vakuumexsikkator über Schwefelsäure getrocknet (Fraktion 1 a). In derselben Weise wurden aus dem jedesmal an der Wand des Kolbens abgesetzten Saponin noch 5 Fraktionen (1 b bis $1 \mathrm{f}$ ) gewonnen. Der Rückstand in dem Kolben (etwa 10\% des verwendeten Saponins) wurde in absolutem Alkohol gelöst und aus der filtrierten Lösung das Saponin mit Aether gefällt (Fraktion $1 \mathrm{~g}$ ).

Für das spezifische Drehungsvermögen in wässeriger Lösung, $[\iota]_{D}^{\prime \prime \prime}$, ergab sich bei der Fraktion $1 \mathrm{a}-9,8^{\circ}, 1 \mathrm{~b}-11,4^{\circ}, 1 \mathrm{c}-11,6^{\circ}$, $1 \mathrm{~d}-12,3^{0}$, 1 e $-12,8^{\circ}$, $1 \mathrm{f}-13,9^{\circ}, 1 \mathrm{~g}-15,9^{\circ}$.

Die Fraktionierung hatte daher nicht zu einer einheitlichen oder annähernd einheitlichen Substanz geführt. Auch die Fraktionen $1 \mathrm{~b}$ und $1 \mathrm{c}$, mit fast übereinstimmenden Drehungen, können durch neues Fraktionieren in zwei Fraktionen mit um ca. 2,50 verschiedener Drehung zerlegt werden.

Für die Fraktion $\mathbf{l ~ b}$ und $\mathbf{l} \mathrm{g}$ wurden aus einigen, genügend stimmenden Elementaranalysen die folgenden Zusammensetzungen berechnet. Darunter befinden sich zwei Glieder der K o b e r t'schen Reihel $\left.{ }^{1}\right)\left(\mathrm{C}_{10} \mathrm{H}_{2 n}{ }_{8} \mathrm{O}_{4}\right)$ mit übereinstimmender Zusammensetzung.

Die Fraktion $1 \mathrm{~b}$ enthält 8,06\% $\mathrm{H} ; 58,01 \% \mathrm{C} ; 33,93 \% \mathrm{O}$. $\mathrm{C}_{23} \mathrm{H}_{38} \mathrm{O}_{10}$ verlangt $8,08 \% \mathrm{H} ; 58,19 \% \mathrm{C} ; 33,73 \% \mathrm{O}$.

Die Fraktion $1 \mathrm{~g}$ enthält $7,89 \% \mathrm{H} ; 57,40 \% \mathrm{C} ; 34,71 \% 0$. $\mathrm{C}_{22} \mathrm{H}_{36} \mathrm{O}_{10}$ verlangt $7,88 \% \mathrm{H} ; 57,35 \% \mathrm{C} ; 34,77 \% 0$.

\section{Spaltung der Saponine durch Săuro.}

Beim Erhitzen der Saponinlösungen mit Säure tritt leicht Spaltung ein in Zucker und eine in Wasser unlösliche Substanz; es tritt dabei ein eigentümlicher Geruch auf.

Völlige Spaltung war nicht so leicht zu erreichen. Nach zweistündigem Erhitzen im siedenden Wasserbade mit Schwefelsäure von $2 \%$ war kein Saponin mehr gelöst; jedoch konnte aus dem Niederschlage durch Erhitzen mit stärkerer Säure noch Zucker abgespalten werden. Erst nach sechsstündigem Erhitzen mit

1) Arb. Dorpat, Pharm. Institut 6, 29 (189I). 
Schwofelsäure von $4 \%$ war so gut wie völlige Spaltung in Zucker und Sapogenin eingetreten.

Zur Gewinnung des abgespaltenen Zuckers wurde, nach dem Abfiltrieren des Sapogenins, das Filtrat mit Baryumkarbonat gekocht; hierauf filtriert und das Filtrat eingedampft. Der braune, hygroskopische Rückstand enthält alsdann den Zucker.

Der Zucker der Fraktionen 1 und 3 enthält Pentose; nzch dem Destillieren mit Salzsäure von $12,5 \%$ gab das Destillat mit Anilinacetat die Furfurolreaktion. Mit Phloroglucin und Salzsäure gab das Destillat, spektroskopisch, die Methylpentosereaktion von $\mathrm{O} \mathrm{sh} \mathrm{im} \mathrm{a} \mathrm{und} \mathrm{Tolle} \mathrm{ns} \mathrm{s}^{1}$.

Auch gaben die Zucker die Pentosereaktionen von $W$ h e e l e r und $\mathrm{T}$ oll $\mathrm{en} \mathrm{s}^{2}$ ) und von $\mathrm{B}$ i a $\mathbf{l}^{3}$ ), jedoch nur spektroskopisch, weil die Pentosefarben durch die Farben der anderen Zucker, besonders der Methylpentosen, verdeckt wurden. In der Fraktion 4 ließ sich wohl Pentose aber keine Methylpentose nachweisen.

In den verschiedenen Fraktionen konnte keine Fruktose und Mannose nachgewiesen werden.

Die mikroskopische Vergleichung der Osazone mit den Osazonen einiger reinen Zucker (Glykose, Galaktose, Arabinose, Xylose und Rhamnose) macht es wahrscheinlich, daß die Fraktionen 1, 3 und 4 Glykose enthalten, die Fraktion 4 daneben noch Arabinose. Das Osazon der Fraktionen 1 und 3 enthält außer den Nadeln des Glykosazons noch sechseckige Täfelchen (bei der Fraktion 3 mit abgerundeten Ecken), welche nicht mit den Osazonen der oben genannten reinen Zucker übereinstimmen und vielleicht von einer Methylpentose herrühren.

Durch die Gärungsprobe wurde die Anwesenheit einer Hexose angezeigt.

Zur quantitativen Bestimmung der Spaltungsprodukte wurde das Sapogenin bei $110^{\circ}$ bis zum konstanten Gewichte getrocknet. Der Zucker wurde nach der S c h o o r l'schen Methoded) mit zwei Minuten Kochzeit als Glykose bestimmt.

Es wurde gefunden, jedesmal aus zwei genügend übereinstimmenden Analysen berechnet, für die Fraktion 1 38,6\% Sapogenin und 60,3\% Zucker als Glykose, für die Fraktion 3 54,0\% Sapogenin und 46,1\% Zucker. Die Fraktion 4 enthielt $61 \%$ Sapo-

1) Ber. d. d. chem. Ges. 34, 1425 (1901).

2) Ann. d. Chem. 254, 329 (1889).

3) Deutsche med. Wochenschr. 28, 253 (1902).

4) Ned. Tijdsch. v. Pharm., Chem. on Tox. 11, 209 (1899). 
genin. Der Zucker wurde nicht bestimmt, weil er für die qualitative Prüfung verwendet war.

Die Pentose (Methylpentose) der Fraktionen 1 und 3 wurde bestimmt nach der Methode von K r üger und Tollensis).

Der Zucker aus 0,9416 g der Fraktion 1 gab 0,2422 g Furfurol-(Methylfurfurol-)phloroglucid, übereinstimmend mit 0,255 g Pentose $=27,2 \%$ oder mit $0,301 \mathrm{~g}$ Methylpentose $^{2}$ ) $=32,0 \%$.

Der Zucker aus 0,9143 g der Fraktion 3 gab 0,1632 g Furfurol-(Methylfurfurol-)phloroglucid, übereinstimmend mit $0,160 \mathrm{~g}$ Pentose $=17,5 \%$ oder mit 0,209 g Methylpentose $=22,9 \%$.

Das Furfurol- und das Methylfurfurolphloroglucid wurden nicht gesondert bestimmt. Da der Absorptionsstreifen der Zucker bei der Reaktion von Wh e ele $\mathrm{r}$ und Tollen s sehr schwach ist im Vergleiche mit demjenigen reiner Pentose, so ist der Pentosegehalt wahrscheinlich nur klein.

Die Sapogen in e wurden noch einmal (nach Auflösen in ein wenig Alkohol) mit Schwefelsäure von 5\% 6 Stunden im siedenden Wasserbade erhitzt; es wurde hierbei noch eine geringe Menge Zucker abgespalten. Beim Wiederholen dieser Operation wurde dagegen kein Zucker mehr abgespalten. Die braunen Sapogenine wurden durch Kochen in weingeistiger Lösung mit Blutkohle fast vollkommen entfärbt.

Sie lösten sich leicht in absolutem Alkohol, kaum in Aether. Beim Erwärmen mit verdünntem Alkali lösten sie sich kaum; wurde jedoch der verdünnten alkoholischen Lösung verdünntes Alkali hinzugefügt und nachher viel Wasser, so blieb die Lösung fast klar. Durch Säure wurden die Sapogenine wieder gefällt. Sie wurden nicht krystallinisch erhalten.

Die Sapogenine stimmen nicht ganz in ihren Eigenschaften überein. Beim Erhitzen bräunen sich die Sapogenine der Fraktionen 1 und 3 schon unter dem Schmelzpunkte; das erste schmilzt bei $286^{\circ}$ (nicht korrigiert) zu einer braunen Flüssigkeit, das andere bei $280^{\circ}$. Das Sapogenin der Fraktion 4 schmilzt orst bei $297^{\circ}$ zu einer lichtgelben Flüssigkeit. Einige Grade unter diesen Temperaturen fangen die Sapogenine schon zu erweichen an.

Für das spezifische Drehungsvermögen, gelöst in absolutem Alkohol, $[u]_{D}^{20}$, wurde gefunden beim Sapogenin der Fraktion 1 $+66^{\circ}$, bei demjenigen der Fraktion $3+59^{\circ}$. Das Sapogenin der Fraktion 1 enthält (Mittel aus zwei Analysen) 10,2\% H; 75,7\% C;

1) Zeitschr. f. angew. Chom., Jahrg. 1896, S. 40.

2) E lett und T ollens, Ber. d. d. chein. (fes. 38, 492 (1905). 
14,1\% 0. Das Sapogenin der Fraktion 3 enthält 9,9\% H; 74,1\% C; $16,0 \% 0$.

\section{Spaltung des Saponins durch Alkali.}

Beim Erhitzen im siedenden Wasserbade einer konzentrierten Lösung der Fraktion 1 in Normal-Natronlauge bildet sich allmählich ein farbloser krystallinischer Niederschlag, indem sich die Lösung bräunt ${ }^{1}$ ). Nach 11/2 Stunden, wenn sich der Niederschlag nicht mehr vermehrte, wurde er abfiltriert und mit Normal-Natronlauge nachgewaschen. Er löst sich leicht in Wasser. Aus dieser Lösung wurde mit Salzsäure in kleinem Ueberschusse ein voluminöser Niederschlag erhalten, welcher abfiltriert wurde. Da derselbe sich beim Auswaschen mit Wasser löste, wurde er mit einer verdünnten Kochsalzlösung bis zur Beseitigung der Säure ausgewaschen. Um alsdann das Kochsalz zu entfernen, wurde der getrocknete Niederschlag mit Spiritus von $25 \%$ ausgezogen, in welchem sich das Kochsalz löst, wogegen die Substanz zurückbleibt. Diese Substanz löst sich im getrockneten Zustande nicht in Wasser; gemischt mit einem Teile der (nicht hämolytischen) Fraktion 1 löst sie sich jedoch klar. In dieser Weise gelöst, ist sie stark hämolytisch. Das Blutgemisch 1:100 wurde durch diese Substanz $1: 150000$ in 24 Stunden völlig hämolysiert; mit einer Lösung $1: 200000$ trat in dieser Zeit teilweise, mit einer Lösung $1: 250000$, eine geringe Hämolyse ein.

Diese hämolytische Substanz ist amorph; sie gibt aber eine krystallinische Kaliumverbindung. Diese wird erhalten, wenn man die alkoholische Lösung mit viel Normal-Kalilauge mischt, und sie nachher zur Beseitigung des Alkohols langsam einengt. Es bilden sich dann wetzsteinförmige Täfelchen mit gerader Auslöschung des polarisierten Lichtes, die größtenteils zu Drusen vereint sind.

Für das spezifische Drehungsvermögen in absolutem Alkohol wurde $+2,0^{\circ}$ gefunden.

Bei der Spaltung mit Säure gab diese Substanz 60,9\% Sapogenin und $39,3 \%$ Zucker (als Glykose bestimmt nach der Methode von $\mathrm{Sch}$ o o r l). Der $\mathrm{Z} \mathrm{u} \mathrm{c} \mathrm{k} \mathrm{e} \mathrm{r} \mathrm{enthält} \mathrm{Methylpentose,} \mathrm{Pentose}$ und Hexose, jedoch hauptsächlich wohl Methylpentose, da das Osazon fast ganz aus sechseckigen Täfelchen (mit abgerundeten Ecken) besteht, wahrscheinlich von einer Methylpentose herrührend. Daneben finden sich auch einige Nadeln wahrscheinlich des Glykos-

1) Die Substanzen, welche beim Erhitzen nit Natronlauge gelöst bleiben, sind nicht untersucht. 
azons. Nach der Gärungsprobe enthält der Zucker weniger als 5\% Hexose. Dieser Gehalt ist so klein, daß die Hexose vielleicht nur ein Spaltungsprodukt eines beigemischten Glykosids und nicht der Hauptsubstanz ist.

Das S a p o ge $\mathbf{n}$ i n, durch Kochen in alkoholischer Lösung mit Blutkohle entfärbt, war amorph. Die Kaliumverbindung in derselben Weise dargestellt, wie diejenige der hämolytischen Substanz, war jedoch krystallinisch. Sie krystallisierte in Nadeln mit gerader Auslöschung.

Das Sapogenin schmilzt bei $297^{\circ}$ (nicht korrigiert) zu einer gelblichen Flüssigkeit. Bei derselben Temperatur schmilzt auch das aus der Kaliumverbindung wieder hergestellte Sapogenin. Einige Grade unter dieser Temperatur erweicht es schon.

Die spezifische Drehung in absolutem Alkohol betrug $+65^{\circ}$; in dieser Hinsicht stimmt das Sapogenin fast überein mit demjenigen der Fraktion 1 (gefunden $+66^{\circ}$ ). Dieses Sapogenin schmilzt jedoch um ca. $10^{\circ}$ niedriger; wahrscheinlich ist es dieselbe Substanz, aber ein wenig verunreinigt.

Die Zusammensetzung der hämolytischen Substanz ist derjenigen der Fraktion 4 ähnlich. Beide enthalten etwa $61 \%$ Sapogenin. Die Sapogenine, welche denselben Schmelzpunkt haben und auch in einigen anderen Eigenschaften übereinstimmen, sind wohl identisch. Die Zucker sind jedoch verschieden; der Zucker der hämolytischen Substanz enthält vorwiegend Methylpentose, derjenige der Fraktion 4 enthält keine Methylpentose (wahrscheinlich Glykose und Arabinose).

Wird eine Lösung der Fraktion 1 in Normal-Alkali 24 Stunden bei gewöhnlicher Temperatur beiseite gesetzt, so tritt auch schon teilweise Spaltung ein. Nach dem Neutralisieren mit Schwefelsäure kann aus der Lösung durch Sättigung mit Magnesiumsulfat ein Saponin gefällt werden, dem hämolytische Substanz beigemischt ist. Die filtrierte Magnesiumsulfatlösung enthält kein Saponin mehr, jedoch den abgespaltenen Zucker. Dieser Zucker ist wahrscheinlich zusammengesetzt; beim Kochen mit Säure tritt Inversion ein. Eine derartige Magnesiumsulfatlösung hatte nach dem Verdünnen mit $1 / 5$ Volum Wasser im $2 \mathrm{dm}$-Rohre eine Drehung von $-0,20^{\circ}$. Dieselbe Lösung zeigte nach einstündigem Erhitzen im siedenden Wasserbade mit $1 / 5$ Volum Salzsäure von $25 \%$ eine Drehung von $+0,15^{\circ}$.

Auch teilweise Spaltung mit Säure macht das Saponin hämolytisch. Eine Lösung der Fraktion 1 wurde mit verdünnter Schwefel- 
säure erhitzt bis die Lösung sich trübte. Jass aus dieser Lösung abgeschiedene Saponin war hämolytisch (etwa 1:10 000).

Das Sapogenin, durch völlige Spaltung mit Säure èrhalten, war, mittels der Fraktion 1 gelöst, nicht hämolytisch.

\section{Zusammenfassung.}

Aus den Trevesiablättern wurde ein Rohsaponin dargestellt; es wurde dabei die Eigensohaft benutzt, daß das Saponin völlig mit Ammoniumsulfat (auch mit Magnesiumsulfat) ausgesalzen werden kann.

Das hämolytische Rohsaponin wurde durch Behandlung mit Magnesiumoxydhydrat in zwei Fraktionen zerlegt, das kaum hämolytische gelbe Saponin und das grüne Saponin, welches stärker hämolytisch war als das Rohsaponin.

Das grüne Saponin ist nicht weiter untersucht. Das gelbe Saponin ist durchaus keine einheitliche Substanz. Durch eine Reihe von Operationen, unter denen wiederholte fraktionierte Fällung aus Alkohol mit Aether die wichtigste war, wurden hieraus der Reihe nach die glykosidischen Fraktionen 1, 2, 3 und 4 erhalten.

Die Fraktion 4 wurde krystallisiert erhalten.

Die Fraktionen 1, 2 und 3 waren amorph. Ihre Eigenschaften sind sehr verschieden. Es sind Gemische, welche ich nicht in einfache Substanzen zerlegen konnte. Das Fraktionieren der Fraktion 1 aus Alkohol führte nicht zu gleichwertigen Fraktionen.

Mit Säure wurden die Fraktionen in Zucker und Sapogenin gespalten. Die Fraktionen 1, 2 und 3 enthalten sowohl Hexose als Pentose und Methylpentose. Die Fraktion 1 enthält mehr Zucker als die Fraktion 3. Den größten Sapogenin- und deshalb den kleinsten Zuckergehalt hat die Fraktion 4; sie enthält keine Methylpentose (wahrscheinlich Glykose und Arabinose).

Auch mit Alkali wird das Saponin zersetzt. Der Zucker wird teilweise (vielleicht als zusammengesetzter Zucker) abgespalten, indem sich u. a. eine neue Saponinsubstanz mit stark hämolytischer Wirkung bildet. Der Zucker dieses Glykosids enthält vorwiegend Methylpentose.

Pharmaz.-chem. Laboratorium der Universität Utrecht. 